\title{
ANALYSIS OF FM DEMODULATOR OUTPUT NOISE WITH APPLICATIONS TO FM TELEMETRY
}

\author{
RAJENDRA KUMAR
}

Received 9 December 2005; Revised 10 February 2006; Accepted 20 February 2006

We present an analysis for evaluating the probability density function (pdf) of the noise at the output of the frequency demodulator. It is shown that the noise is non-Gaussian and that for low to medium signal-to-noise power ratios, its pdf differs very significantly from the Gaussian pdf commonly assumed in simplified analysis. These results are very important for analyzing the performance of the PCM/FM type of modulation schemes used in telemetry systems as illustrated in the paper.

Copyright (c) 2006 Rajendra Kumar. This is an open access article distributed under the Creative Commons Attribution License, which permits unrestricted use, distribution, and reproduction in any medium, provided the original work is properly cited.

\section{Introduction}

This paper presents an exact analysis for the probability density function (pdf) of the noise at the output of a frequency demodulator. In the literature $[1,5]$, the FM demodulator output noise is assumed to be Gaussian-distributed at high signal-to-noise power ratio (SNR). At low SNR, it is analyzed in terms of a Gaussian noise and a sequence of impulse functions (clicks) based on the classical theory propounded by Rice [4]. In such an analysis, assuming relatively low bandwidth of the postdemodulator lowpass (LPF) filter, the variance of the total noise power at the filter output is evaluated. The probability distribution of the filter output noise is assumed to be Gaussian. While such an analysis is adequate for the case of analog information signals such as speech, for the case of digital signals, one of the most important characteristics of the noise is its pdf.

This paper presents an exact analysis of the FM demodulator output noise under the assumption of low-modulation index and Gaussian-distributed noise at the demodulator input. It is shown that the pdf is given in terms of hypergeometric function. The derived expression is applicable to all SNRs. At relatively low to medium SNR levels, the pdf of noise differs drastically from the Gaussian pdf with the difference becoming progressively smaller with increasing SNR. At high SNRs (> $25 \mathrm{~dB})$, the difference is relatively small. However, since the typical SNR used in digital telemetry is in the 10-15 $\mathrm{dB}$ range, the nonGaussian distribution is very important in evaluating the probability of bit error. Due 


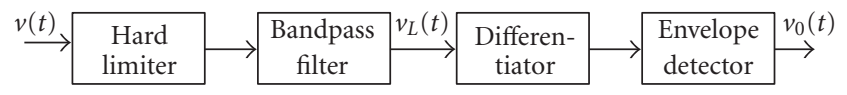

Figure 2.1. Frequency demodulator.

to the analytical difficulties, the analysis does not take into account the effect of lowpass filter following the FM demodulator. However, this effect is expected to be relatively small when the lowpass filter bandwidth is of the order of the IF bandwidth which has been shown to be optimum from earlier simulation results.

\section{Signal model}

The received frequency-modulated RF signal is filtered and down-converted to an intermediate frequency $\omega_{\text {IF }}$ before detection. The frequency-modulated signal at the output of the IF filter is given by

$$
v(t)=A \cos \left(\omega_{\mathrm{IF}} t+\theta_{s}(t)\right)+n(t),
$$

where $\omega_{\mathrm{IF}}$ denotes the IF frequency, $\theta_{s}(t)$ is the signal modulation and is equal to $D_{f}$ $\int_{-\infty}^{t} m(\tau) d \tau$ where $D_{f}$ denotes the frequency modulator sensitivity, and $m(t)$ is the message signal. Note that the derivative of the phase $\theta_{s}(t)$, termed the instantaneous frequency deviation, is equal to $D_{f} m(t)$ thus signifying the term frequency modulation for $v(t)$ in (2.1). The term $n(t)$ represents band-limited "white" noise with one-sided power spectral density equal to $N_{0}$ and is assumed to have a Gaussian distribution. The variance of $n(t)$ is equal to $N_{0} B_{\mathrm{IF}}$ where $B_{\mathrm{IF}}$ is the IF filter equivalent noise bandwidth. The noise term $n(t)$ may be expressed in terms of the following in-phase and quadrature representation:

$$
n(t)=x_{n}(t) \cos \left(\omega_{\mathrm{IF}} t\right)-y_{n}(t) \sin \left(\omega_{\mathrm{IF}} t\right),
$$

where $x_{n}(t)$ and $y_{n}(t)$ are baseband "white" noise processes with (one-sided) power spectral density $2 N_{0}$ and of bandwidth $B_{\mathrm{IF}} / 2$. The processes $x_{n}(t)$ and $y_{n}(t)$ are independent Gaussian and have variance $\sigma^{2}=N_{0} B_{\mathrm{IF}}$.

Figure 2.1 shows the block diagram of a frequency demodulator [1] wherein the signal at the input to the demodulator is specified by (2.1). The bandpass filter of bandwidth $B_{\mathrm{IF}}$ following the hard limiter in the figure selects only the fundamental zone signal while filtering out all the higher-order zone signals.

The fundamental zone signal $v_{L}(t)$ has a constant amplitude $A_{L}$ which is equal to $(4 / \pi)$ times the hard limiter output voltage level, frequency $\omega_{\mathrm{IF}}$, and phase $\theta_{T}(t)$ and is given by

$$
\begin{gathered}
v_{L}(t)=A_{L} \cos \left(\omega_{\mathrm{IF}} t+\theta_{T}(t)\right), \\
\theta_{T}=\theta_{s}+\tan ^{-1}\left\{\frac{R_{n} \sin \left(\theta_{n}-\theta_{s}\right)}{A+R_{n} \cos \left(\theta_{n}-\theta_{s}\right)}\right\},
\end{gathered}
$$


where the dependence of various terms in (2.3b) on time $t$ has been suppressed for notational simplicity, $\theta_{s}(t)$ denotes the desired signal, and $R_{n}(t)$ and $\theta_{n}(t)$ represent the amplitude and phase of the additive noise $n(t)$ with its complex envelope given by

$$
g_{n}(t)=R_{n}(t) e^{j \theta_{n}(t)} ; \quad g_{n}(t)=x_{n}(t)+j y_{n}(t)
$$

The complex envelope of the received signal is similarly given by

$$
g_{s}(t)=A e^{j \theta_{s}(t)}
$$

Thus the envelope of the derivative of $v_{L}(t)$ denoted by $v_{0}(t)$ in Figure 2.1 is equal to

$$
v_{0}(t)=A_{L}\left[\omega_{\mathrm{IF}}+\frac{d \theta_{T}(t)}{d t}\right]
$$

under the assumption that $\omega_{\mathrm{IF}}>\max \left|d \theta_{T}(t) / d t\right|$, which is always satisfied in practice. Assuming that $A_{L}$ is equal to 1 without any loss of generality and ignoring the constant term, the output of the frequency demodulator is simply equal to $\left(d \theta_{T} / d t\right)$. Note that Figure 2.1 shows a more conventional implementation of the frequency demodulator and there are other equivalent analog and digital implementations of the same.

In the first instance it is assumed that the frequency modulation index is relatively small which is equivalent to assuming that the phase modulation index defined by $\max \left|\theta_{s}(t)\right|$ is much smaller compared to $1\left(\theta_{s}(t)\right.$ is small compared to $\left.\theta_{n}(t)\right)$. In this case the noise term in (2.3b) may be approximated as

$$
\phi_{n} \cong \tan ^{-1}\left\{\frac{R_{n} \sin \left(\theta_{n}\right)}{A+R_{n} \cos \left(\theta_{n}\right)}\right\} .
$$

Equivalently one may express $\phi_{n}$ as

$$
\begin{gathered}
\phi_{n}=\tan ^{-1}\left[\frac{y_{n}}{A_{c}+x_{n}}\right], \\
x_{n}=R_{n} \cos \left(\theta_{n}\right) ; \quad y_{n}=R_{n} \sin \left(\theta_{n}\right) .
\end{gathered}
$$

Differentiation of (2.8a) with respect to the time $t$ yields the following expression for the FM demodulator output noise:

$$
\dot{\phi}_{n}=\frac{\left(A+x_{n}\right) \dot{y}_{n}-\dot{x}_{n} y_{n}}{\left[\left(A+x_{n}\right)^{2}+y_{n}^{2}\right]} .
$$

In the following, an expression for the pdf of $\dot{\phi}_{n}$ is derived, which is valid for the complete range of SNR. 


\section{Demodulator output noise distribution}

To derive the requisite pdf, the set of random variables (RV) $X_{1}, X_{2}, X_{3}$, and $X_{4}$ are defined for notational convenience by

$$
X_{1}=\left(A+x_{n}\right) ; \quad X_{2}=y_{n} ; \quad X_{3}=\dot{x}_{n} ; \quad X_{4}=\dot{y}_{n},
$$

then the desired RV $\dot{\phi}_{n}$ may be expressed in terms of RVs $X_{1}, X_{2}, X_{3}$, and $X_{4}$ as

$$
\dot{\phi}_{n}=\frac{X_{1} X_{4}-X_{2} X_{3}}{X_{1}^{2}+X_{2}^{2}}
$$

From the discussion of the in-phase and quadrature representation of $n(t)$ in (2.2), it follows that $X_{1}$ and $X_{2}$ are independent and Gaussian-distributed with variance $\sigma^{2}=N_{0} B_{\text {IF }}$. Therefore $X_{3}$ and $X_{4}$ are also Gaussian and probabilistically independent. The power spectral density (one-sided) of $\dot{x}_{n}(t)$ is given by $P_{\dot{x}_{n}}(f)=(2 \pi f)^{2} P_{x_{n}}(f)$ where $P_{x_{n}}(f)$ is the power spectral density (PSD) of $x_{n}(t)$, and thus the variance of $\dot{x}_{n}(t)$ denoted by $\sigma_{d}^{2}$ is given by

$$
\sigma_{d}^{2}=\int_{0}^{\infty} 4 \pi^{2} f^{2} P_{x_{n}}(f) d f
$$

For the case when the IF filter is assumed to be ideal with bandwidth $B_{\mathrm{IF}}=2 B, \sigma_{d}^{2}$ may be evaluated to be

$$
\sigma_{d}^{2}=(2 \pi B)^{2} \frac{\sigma^{2}}{3}
$$

Note however that (3.3) is more general and applies to any filter shape. Similarly the variance of $\dot{y}_{n}(t)$ is also given by $\sigma_{d}^{2}$. It easily follows as is well known that the crosscorrelation function of $y_{n}(t)$ and $\dot{y}_{n}(t)$ denoted by $R_{y_{n}} \dot{y}_{n}(\tau) \equiv \overline{y_{n}(t) \cdot \dot{y}_{n}(t+\tau)}$ is given by

$$
R_{y_{n} \dot{y}_{n}}(\tau)=\frac{d R(\tau)}{d \tau}
$$

and thus the variables $y_{n}$ and $\dot{y}_{n}$ are uncorrelated if $R(\tau)$ has a maximum at $\tau=0$ which is true for most practical filters including the ideal filter case. Because of the Gaussian distribution of the two variables, it follows that they are also statistically independent. Similarly the variables $x_{n}$ and $\dot{x}_{n}$ are also independent. In summary, $X_{1}, X_{2}, X_{3}, X_{4}$ are statistically independent and Gaussian-distributed random variables. In order to evaluate the pdf (probability density function) of $\dot{\phi}_{n}$, a set of intermediate random variables 
$Y_{1}, Y_{2}, Y_{3}, Y_{4}$ is defined by

$$
Y_{1}=X_{1} X_{4}-X_{2} X_{3} ; \quad Y_{2}=X_{1}^{2}+X_{2}^{2} ; \quad Y_{3}=X_{1} X_{4} ; \quad Y_{4}=X_{1}^{2}
$$

To evaluate the pdf of the random vector $\underline{Y}=\left[\begin{array}{llll}Y_{1} & Y_{2} & Y_{3} & Y_{4}\end{array}\right]$; the set of (3.6) is solved for the value of the random vector

$$
\underline{X}=\underline{x}=\left[\begin{array}{llll}
x_{1} & x_{2} & x_{3} & x_{4}
\end{array}\right] \quad \text { with } \underline{Y}=\underline{y}=\left[\begin{array}{llll}
y_{1} & y_{2} & y_{3} & y_{4}
\end{array}\right]
$$

The desired solutions of (3.6) are given by

$$
\underline{x}=\underline{g}^{-1}(\underline{y})=\left(\begin{array}{c} 
\pm \sqrt{y_{4}} \\
\pm \sqrt{\left(y_{2}-y_{4}\right)} \\
\pm \frac{\left(y_{3}-y_{1}\right)}{\sqrt{\left(y_{2}-y_{4}\right)}} \\
\pm \frac{y_{3}}{\sqrt{y_{4}}}
\end{array}\right) .
$$

In (3.8) $x_{1}$ and $x_{4}$ (resp., $x_{2}$ and $x_{3}$ ) have same sign. Thus there are four possible solutions for (3.8). Considering first the solution with all signs positive, the Jacobian $J$ of the set of (3.8) may be shown to be

$$
J \equiv\left|\frac{\partial \underline{x}}{\partial \underline{y}}\right|=\frac{-1}{\left[4 y_{4}\left(y_{2}-y_{4}\right)\right]} .
$$

In (3.9), $|A|$ denotes the determinant of any matrix $A$. As the random variables $X_{1}, X_{2}$, $X_{3}$, and $X_{4}$ are statistically independent, the joint pdf of the random vector $\underline{X}$ denoted by $f_{\underline{X}}(\underline{x})$ is given by

$$
f_{\underline{X}}(\underline{x})=f_{X_{1}}\left(x_{1}\right) f_{X_{2}}\left(x_{2}\right) f_{X_{3}}\left(x_{3}\right) f_{X_{4}}\left(x_{4}\right)
$$

and the component (corresponding to the selected solution from (3.8)) of the pdf of the random vector $\underline{Y}$ denoted by $f_{\underline{Y}}^{1}(y)$ is given by

$$
f_{\underline{Y}}^{1}(\underline{y})=|J| \cdot f_{X_{1}}\left(g_{1}^{-1}(\underline{y})\right) \cdot f_{X_{2}}\left(g_{2}^{-1}(\underline{y})\right) \cdot f_{X_{3}}\left(g_{3}^{-1}\left(y_{3}\right)\right) \cdot f_{X_{4}}\left(g_{4}^{-1}\left(y_{4}\right)\right) .
$$

In the subsequent development $f_{\underline{Y}}^{1}(y)$ and its integral with respect to components of $y$ are referred to as pdf and marginal pdf's, respectively. The actual pdf is the sum of such components evaluated for all four solutions in (3.8). In (3.11), $g_{1}, g_{2}, g_{3}$, and $g_{4}$ represent the components of the vector function $g(y)$. The various functions appearing in (3.11) 
are given by

$$
\begin{gathered}
f_{X_{1}}\left(g_{1}^{-1}(\underline{y})\right)=\frac{1}{\sqrt{2 \pi} \sigma} \exp \left\{-\frac{\left(\sqrt{y_{4}}-A\right)^{2}}{2 \sigma^{2}}\right\}, \\
f_{X_{2}}\left(g_{2}^{-1}(\underline{y})\right)=\frac{1}{\sqrt{2 \pi} \sigma} \exp \left\{-\frac{\left(\sqrt{y_{2}-y_{4}}\right)^{2}}{2 \sigma^{2}}\right\}, \\
f_{X_{3}}\left(g_{3}^{-1}(\underline{y})\right)=\frac{1}{\sqrt{2 \pi} \sigma_{d}} \exp \left\{-\frac{\left(y_{3}-y_{1}\right)^{2}}{\left[2\left(y_{2}-y_{4}\right) \sigma_{d}^{2}\right]}\right\}, \\
f_{X_{4}}\left(g_{4}^{-1}(\underline{y})\right)=\frac{1}{\sqrt{2 \pi} \sigma_{d}} \exp \left\{-\frac{y_{3}^{2}}{2 y_{4} \sigma_{d}^{2}}\right\},
\end{gathered}
$$

where $\sigma^{2}$ and $\sigma_{d}^{2}$ denote the variances of random variables $X_{1}$ and $X_{3}$ (resp., $X_{2}$ and $X_{4}$ ). Now the product of the last two terms in (3.12) may be written in the following form:

$$
\begin{gathered}
f_{X_{3}}\left(g_{3}^{-1}(\underline{y})\right) \cdot f_{X_{4}}\left(g_{4}^{-1}(\underline{y})\right)=\frac{1}{2 \pi \sigma_{d}^{2}} \exp \left\{-\frac{y_{1}^{2}}{2 y_{2} \sigma_{d}^{2}}\right\} \cdot \exp \left\{-\frac{\left(y_{3}-m\right)^{2}}{2 \sigma_{0}^{2}}\right\}, \\
m \equiv \frac{y_{1} y_{4}}{y_{2}} ; \quad \sigma_{0}^{2}=\frac{y_{4}}{y_{2}}\left(y_{2}-y_{4}\right) \sigma_{d}^{2} .
\end{gathered}
$$

Substitution of (3.12) and (3.9) in (3.11) yields the joint pdf of the random vector $\underline{Y}$. The desired joint pdf of the random variables $Y_{1}, Y_{2}$ is obtained by integrating the joint pdf $f_{\underline{Y}}^{1}(y)$ with respect to $y_{3}, y_{4}$. Since only the last two product terms in (3.11) are functions of $y_{3}$, the marginal pdf of $Y_{1}, Y_{2}, Y_{4}$ is given by

$$
\begin{aligned}
f_{Y_{1}, Y_{2}, Y_{4}}^{1}\left(y_{1}, y_{2}, y_{4}\right)= & \frac{1}{4 y_{4}\left(y_{2}-y_{4}\right)} f_{X_{1}}\left(g_{1}^{-1}(\underline{y})\right) f_{X_{2}}\left(g_{2}^{-1}(\underline{y})\right) \\
& \times \int_{y_{3}=-\infty}^{\infty} f_{X_{3}}\left(g_{3}^{-1}(\underline{y})\right) f_{X_{4}}\left(g_{4}^{-1}(\underline{y})\right) d y_{3} .
\end{aligned}
$$

The integral in (3.15) may be easily evaluated by substitution from (3.13) and is given by

$$
\int_{y_{3}=-\infty}^{\infty} f_{X_{3}}\left(g_{3}^{-1}(\underline{y})\right) f_{X_{4}}\left(g_{4}^{-1}(\underline{y})\right) d y_{3}=\frac{1}{\sqrt{2 \pi} \sigma_{d}}\left\{\frac{y_{4}\left(y_{2}-y_{4}\right)}{y_{2}}\right\}^{1 / 2} \exp \left\{-\frac{y_{1}^{2}}{2 y_{2} \sigma_{d}^{2}}\right\} .
$$

With the substitution for the integral from (3.16) and the expressions for $f_{X_{1}}(\cdot)$ and $f_{X_{2}}(\cdot)$ from (3.12a), (3.12b) in (3.15), the desired marginal pdf of $Y_{1}, Y_{2}, Y_{4}$ is given by

$$
\begin{aligned}
f_{Y_{1}, Y_{2}, Y_{4}}\left(y_{1}, y_{2}, y_{4}\right)= & \frac{1}{4 \sqrt{2 \pi} \sigma_{d}} \exp \left\{-\frac{y_{1}^{2}}{2 y_{2} \sigma_{d}^{2}}\right\} \frac{1}{2 \pi \sigma^{2} y_{2}^{3 / 2}} \exp \left\{-\frac{\left(A^{2}+y_{2}\right)}{2 \sigma^{2}}\right\} \\
& \times\left[\frac{y_{4}}{y_{2}}\right]^{(-1 / 2)}\left[1-\frac{y_{4}}{y_{2}}\right]^{(-1 / 2)} \exp \left\{\frac{A \sqrt{y_{4}}}{\sigma^{2}}\right\} .
\end{aligned}
$$


Finally the marginal pdf of $Y_{1}, Y_{2}$ is obtained by integrating the right-hand side of (3.17) with respect to $y_{4}$. Let $I$ represent the following integral:

$$
I=\int_{y_{4}=0}^{y_{2}}\left[\frac{y_{4}}{y_{2}}\right]^{(-1 / 2)}\left[1-\frac{y_{4}}{y_{2}}\right]^{(-1 / 2)} \exp \left\{\frac{A \sqrt{y_{4}}}{\sigma^{2}}\right\} d y_{4} .
$$

With the change of variables $v=\sqrt{\left(y_{4} / y_{2}\right)}$, the integral may be rewritten as

$$
I=2 y_{2} \int_{v=0}^{1}\left(1-v^{2}\right)^{-1 / 2} \exp (\beta v) d v ; \quad \beta \equiv \frac{A}{\sigma^{2}} \sqrt{y_{2}}
$$

Using the identity of $[2,(3.389 / 1)]$ reproduced below,

$$
\begin{aligned}
\int_{0}^{1} x^{2 \nu-1}\left(1-x^{2}\right)^{\rho-1} \exp (\mu x) d x \\
=\frac{1}{2} B(\nu, \rho)_{1} F_{2}\left[\nu ; \frac{1}{2}, \nu+\rho ; \frac{\mu^{2}}{4}\right] \\
+\frac{\mu}{2} B\left[\left(\nu+\frac{1}{2}\right), \rho\right]{ }_{1} F_{2}\left[\left(\nu+\frac{1}{2}\right) ; \frac{3}{2},\left(\nu+\rho+\frac{1}{2}\right) ; \frac{\mu^{2}}{4}\right] \quad[\operatorname{Re} \rho>0 ; \operatorname{Re} \nu>0],
\end{aligned}
$$

where ${ }_{1} F_{2}$ represents the hypergeometric function. The application of identity (3.20) with $\rho=1 / 2$ and $\nu=1 / 2$ yields

$$
I=B\left[\frac{1}{2}, \frac{1}{2}\right]{ }_{1} F_{2}\left[\frac{1}{2} ; \frac{1}{2}, 1 ; \frac{\beta^{2}}{4}\right]+\beta B\left[1, \frac{1}{2}\right]{ }_{1} F_{2}\left[1 ; \frac{3}{2}, \frac{3}{2} ; \frac{\beta^{2}}{4}\right],
$$

where $B$ denotes the beta function (Euler's integral of first kind) [2]. Representing the ${ }_{1} F_{2}(\cdot)$ functions in terms of their series expansions, the integral $I$ may be expressed as

$$
I=\sum_{k=0}^{\infty} c_{k} \beta^{k}=\sum_{k=0}^{\infty} c_{k}\left(\frac{A}{\sigma^{2}}\right)^{k}\left(\sqrt{y_{2}}\right)^{k}
$$

where the coefficients $c_{k}$ may be evaluated in terms of the coefficients in the series expansions of the ${ }_{1} F_{2}(\cdot)$ functions appearing in (3.21), such an evaluation is carried out in the subsequent development. Therefore, the marginal pdf of the random variables $Y_{1}, Y_{2}$ is given by

$$
f_{Y_{1}, Y_{2}}^{1}\left(y_{1}, y_{2}\right)=\frac{1}{4 \sqrt{2 \pi} \sigma_{d}} \exp \left\{-\frac{y_{1}^{2}}{2 y_{2} \sigma_{d}^{2}}\right\} \frac{1}{2 \pi \sigma^{2} y_{2}^{1 / 2}} \exp \left\{-\frac{A^{2}}{2 \sigma^{2}}\right\} \times \exp \left\{-\frac{y_{2}}{2 \sigma^{2}}\right\} \sum_{k=0}^{\infty} c_{k} \beta^{k} .
$$

Now the pdf of the desired random variable $Z \equiv Y_{1} / Y_{2}$ is given by [3]

$$
f_{Z}^{1}(z)=\int_{w=-\infty}^{\infty}|w| f_{Y_{1}, Y_{2}}^{1}(z w, w) d w .
$$


Substitution of the joint pdf of $Y_{1}, Y_{2}$ from (3.23) into (3.24) results in the following expression for the pdf of $Z$ :

$$
\begin{aligned}
f_{Z}^{1}(z)= & \frac{1}{4(2 \pi)^{3 / 2} \sigma_{d} \sigma^{2}} \exp \left[-\frac{A^{2}}{2 \sigma^{2}}\right] \sum_{k=0}^{\infty} c_{k}\left(\frac{A}{\sigma^{2}}\right)^{k} \\
& \times \int_{w=0}^{\infty}(\sqrt{w})^{k+1} \exp \left\{-\left[1+\left(\frac{z \sigma}{\sigma_{d}}\right)^{2}\right] \frac{w}{2 \sigma^{2}}\right\} d w .
\end{aligned}
$$

Using the identity in $[2,(3.351)]$ reproduced in (3.26) below,

$$
\int_{0}^{\infty} x^{\nu-1} \exp (-\mu x) d x=\frac{1}{\mu^{\nu}} \Gamma(\nu) \quad[\operatorname{Re} \nu>0 ; \operatorname{Re} \mu>0],
$$

where $\Gamma(\cdot)$ denotes the gamma function, the integral in (3.25) denoted by $I_{2}$ is given by

$$
I_{2}=\left[\frac{2 \sigma^{2}}{1+\left(z \sigma / \sigma_{d}\right)^{2}}\right]^{(k+3) / 2} \Gamma\left[\frac{k+3}{2}\right] .
$$

Substituting for $I_{2}$ from (3.27) into (3.25), the desired pdf $f_{Z}(z)$ may be expressed in the following form:

$$
f_{Z}^{1}(z)=\frac{\sigma / \sigma_{d}}{4(\pi)^{3 / 2}}\left\{\frac{\exp \left(-A^{2} /\left(2 \sigma^{2}\right)\right)}{\left[1+\left(z \sigma / \sigma_{d}\right)^{2}\right]^{3 / 2}}\right\} \sum_{k=0}^{\infty} c_{k} \Gamma\left[\frac{k+3}{2}\right]\left[\frac{2(A / \sigma)^{2}}{1+\left(z \sigma / \sigma_{d}\right)^{2}}\right]^{k / 2} .
$$

Form the definition of the coefficients $c_{k}$ given implicitly by (3.21)-(3.22) and using the series expansion for the hypergeometric functions [2], the expression for $c_{k}$ is given by

$$
\begin{gathered}
c_{2 j}=B\left[\frac{1}{2}, \frac{1}{2}\right] \frac{(1 / 2)_{j}}{(1 / 2)_{j} \cdot(1)_{j}} \frac{1}{j !}\left(\frac{1}{4}\right)^{j} ; \quad j=1,2, \ldots ; \\
c_{2 j+1}=B\left[1, \frac{1}{2}\right] \frac{(1)_{j}}{(3 / 2)_{j} \cdot(3 / 2)_{j}} \frac{1}{j !}\left(\frac{1}{4}\right)^{j} ; \quad j=1,2, \ldots ; \\
c_{0}=B\left[\frac{1}{2}, \frac{1}{2}\right] ; \quad c_{1}=B\left[1, \frac{1}{2}\right] ; \\
(\alpha)_{j} \equiv(\alpha)(\alpha+1) \cdots(\alpha+j-1) ; \quad j \geq 1 ;(\alpha)_{0}=1 \text { for any } \alpha .
\end{gathered}
$$

Therefore, substituting the expansion for the gamma function, one obtains the following expression for the summand in (3.28):

$$
\begin{aligned}
& c_{k} \Gamma\left[\frac{k+3}{2}\right] w^{k / 2}=B\left[\frac{1}{2}, \frac{1}{2}\right] \Gamma\left[\frac{3}{2}\right] \frac{(1 / 2)_{j} \cdot(3 / 2)_{j}}{(1 / 2)_{j} \cdot(1)_{j}} \frac{1}{j !}\left(\frac{w}{4}\right)^{j} ; \quad k=2 j ; \\
& c_{k} \Gamma\left[\frac{k+3}{2}\right] w^{k / 2}=B\left[1, \frac{1}{2}\right] \sqrt{w} \frac{(1)_{i} \cdot(2)_{i}}{(3 / 2)_{i} \cdot(3 / 2)_{i}} \frac{1}{i !}\left(\frac{w}{4}\right)^{i} ; \quad k=2 i+1 ;
\end{aligned}
$$


where in (3.30) $w$ has been defined as

$$
w \equiv\left[\frac{2(A / \sigma)^{2}}{1+\left(z \sigma / \sigma_{d}\right)^{2}}\right] .
$$

From (3.30) it follows that

$$
\begin{aligned}
\sum_{k=0}^{\infty} c_{k} \Gamma\left[\frac{k+3}{2}\right] w^{k / 2}= & B\left[\frac{1}{2}, \frac{1}{2}\right] \cdot \Gamma\left[\frac{3}{2}\right]{ }_{2} F_{2}\left[\frac{1}{2}, \frac{3}{2} ; \frac{1}{2}, 1 ; \frac{w}{4}\right] \\
& +B\left[1, \frac{1}{2}\right] \sqrt{w}_{2} F_{2}\left[1,2 ; \frac{3}{2}, \frac{3}{2} ; \frac{w}{4}\right] .
\end{aligned}
$$

Finally the substitution of (3.32) into (3.28) results in the following expression for $f_{Z}(z)$ :

$$
\begin{aligned}
f_{Z}^{1}(z)=\frac{\sigma / \sigma_{d}}{4(\pi)^{3 / 2}}\left\{\frac{\exp \left[-A^{2} /\left(2 \sigma^{2}\right)\right]}{\left[1+\left(z \sigma / \sigma_{d}\right)^{2}\right]^{3 / 2}}\right\} \\
\times\left\{B\left[\frac{1}{2}, \frac{1}{2}\right] \cdot \Gamma\left[\frac{3}{2}\right]{ }_{2} F_{2}\left[\frac{1}{2}, \frac{3}{2} ; \frac{1}{2}, 1 ; \widetilde{w}\right]\right. \\
\left.+2 B\left[1, \frac{1}{2}\right] \sqrt{\widetilde{w}}_{2} F_{2}\left[1,2 ; \frac{3}{2}, \frac{3}{2} ; \widetilde{w}\right]\right\} ; \\
\widetilde{w} \equiv\left[\frac{\left[A^{2} /\left(2 \sigma^{2}\right)\right]}{1+\left(z \sigma / \sigma_{d}\right)^{2}}\right] .
\end{aligned}
$$

It may be shown in a manner similar to the derivation of $f_{Z}^{1}(z)$ that the component of the pdf $f_{Z}(z)$ corresponding to the negative sign for $x_{1}$ and $x_{4}$ in (3.8) is obtained by replacing the positive sign associated with the second term in (3.33a) by negative sign. The solutions corresponding to the other two solutions are identical to the first two solutions. Hence the pdf $f_{Z}(z)$ is equal to four times the first term in (3.33a), that is,

$$
f_{Z}(z)=\frac{\sigma / \sigma_{d}}{2(\pi)^{3 / 2}}\left\{\frac{\exp \left[-A^{2} /\left(2 \sigma^{2}\right)\right]}{\left[1+\left(z \sigma / \sigma_{d}\right)^{2}\right]^{3 / 2}}\right\} \times B\left[\frac{1}{2}, \frac{1}{2}\right] \cdot \Gamma\left[\frac{1}{2}\right]{ }_{1} F_{1}\left[\frac{3}{2} ; 1 ; \widetilde{w}\right],
$$

where using the definition of the hypergeometric functions, the ${ }_{2} F_{2}(\cdot)$ function has been simplified to the ${ }_{1} F_{1}(\cdot)$ function and the $\Gamma(3 / 2)$ has been replaced by $(1 / 2) \Gamma(1 / 2)$. Equation (3.34) with (3.33b) represent the final desired result. Finally substituting the values of the beta and gamma functions, $B[(1 / 2),(1 / 2)]=\pi$ and $\Gamma[1 / 2]=\sqrt{\pi}$, the expression for the pdf $f_{Z}(z)$ simplifies to

$$
f_{Z}(z)=\frac{\sigma / \sigma_{d}}{2}\left\{\frac{\exp \left[-A^{2} /\left(2 \sigma^{2}\right)\right]}{\left[1+\left(z \sigma / \sigma_{d}\right)^{2}\right]^{3 / 2}}\right\}{ }_{1} F_{1}\left[\frac{3}{2} ; 1 ; \widetilde{w}\right], \quad \widetilde{w} \equiv\left[\frac{\left[A^{2} /\left(2 \sigma^{2}\right)\right]}{1+\left(z \sigma / \sigma_{d}\right)^{2}}\right] .
$$

More conveniently defining the normalized random variable $\Psi=Z /(2 \pi B)$ in view of (3.4) (frequency fluctuation normalized by the filter bandwidth in $\mathrm{rad} / \mathrm{s}$ ), the $\mathrm{pdf}$ of the 


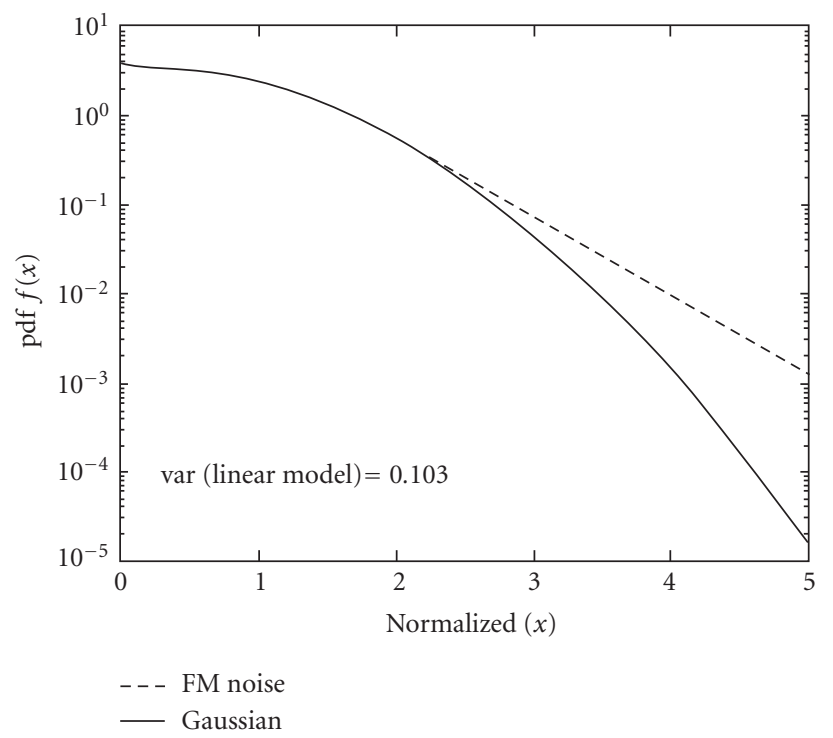

Figure 3.1. FM demodulator output noise $\mathrm{pdf}(\mathrm{SNR}=12 \mathrm{~dB})$.

RV $\Psi$ is given by

$$
f_{\Psi}(\psi)=\frac{\sigma / \sigma_{f}}{2}\left\{\frac{\exp \left[-A^{2} /\left(2 \sigma^{2}\right)\right]}{\left[1+\left(\psi \sigma / \sigma_{f}\right)^{2}\right]^{3 / 2}}\right\}{ }_{1} F_{1}\left[\frac{3}{2} ; 1 ; \tilde{w}\right], \quad \widetilde{w} \equiv\left[\frac{\left[A^{2} /\left(2 \sigma^{2}\right)\right]}{1+\left(\psi \sigma / \sigma_{f}\right)^{2}}\right],
$$

where $\sigma_{f}=\sigma_{d} /(2 \pi B)$ in (3.36) and thus $\sigma_{f}^{2}$ represents the variance of the normalized frequency fluctuations. Note that under the high-SNR condition and ideal filter shape usually treated in the literature, $\sigma_{f}^{2}=\sigma^{2} /\left(3 A^{2}\right) \equiv \sigma_{l}^{2}$.

3.1. Comparison with Gaussian pdf. Figures 3.1-3.2 plot the pdf of the demodulator output noise as computed from (3.36) for the input SNR $=\left(A^{2} / 2 \sigma^{2}\right)$ equal to 12 and $20 \mathrm{~dB}$, respectively, plotted versus $\psi$ normalized by $\sigma_{l}$ where $\sigma_{l}^{2}=\sigma^{2} /\left(3 A^{2}\right)$ is the variance of $\psi$ predicted on the basis of linear Gaussian assumption [1, 5]. As may be observed from these figures, the pdf as computed from (3.36) differs very markedly from its value predicted from the linear theory for SNR up to $15 \mathrm{~dB}$ with lower SNR resulting in higher difference. For an SNR equal to $20 \mathrm{~dB}$ the difference is relatively small. Table 3.1 shows the rms value of the noise $\sigma_{f}$ as computed from (3.36) and its value $\sigma_{l}$ as predicted from linear approximation for various values of SNR.

While the linear theory gives good approximation for the noise variance for SNR greater than or equal to $12 \mathrm{~dB}$, the results in terms of pdf differ significantly at these SNRs. For example, at SNR of $12 \mathrm{~dB}$, at $x=\psi / \sigma_{l}$ equal to 5 , the value of pdf predicted from linear approximation is $1.5 \times 10^{-5}$ compared to $1.1 \times 10^{-4}$ predicted from the results of this paper. Thus the non-Gaussian nature of the noise is of high significance in 


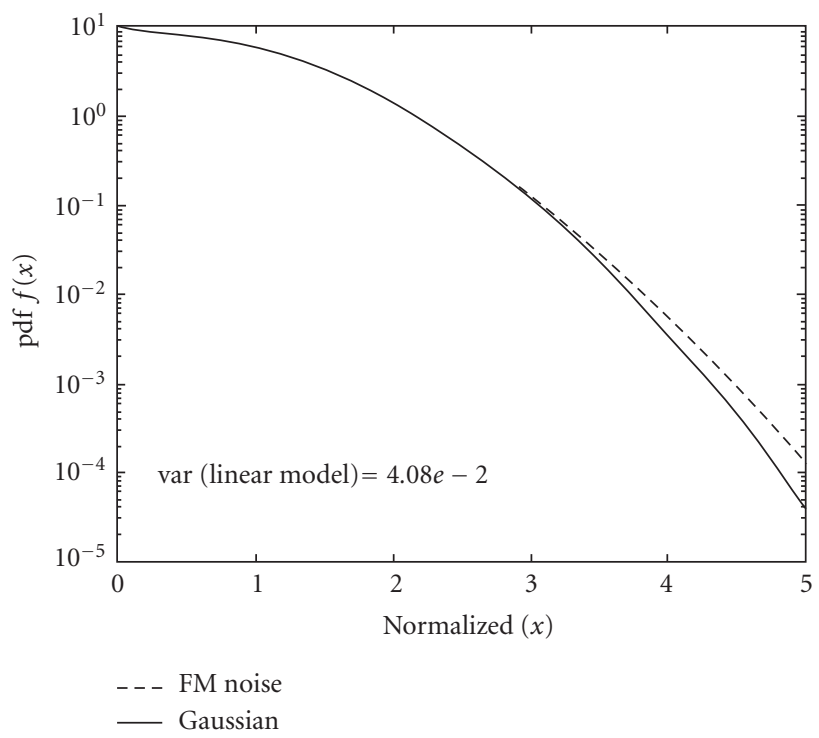

Figure 3.2. FM demodulator output noise $\mathrm{pdf}(\mathrm{SNR}=20 \mathrm{~dB})$.

Table 3.1. Comparison of theory with Gaussian approximation.

\begin{tabular}{c|c|c|c}
\hline SNR $(\mathrm{dB})$ & $\sigma_{l}$ & $\sigma_{f}$ & $20^{\star} \log \left(\sigma_{f} / \sigma_{l}\right)$ \\
\hline 6 & 0.2046 & 0.305 & 3.47 \\
8 & 0.1625 & 0.1895 & 1.34 \\
12 & 0.1025 & 0.1062 & 0.308 \\
15 & 0.0726 & 0.0738 & 0.142 \\
20 & 0.0408 & 0.0410 & 0.042 \\
25 & 0.0230 & 0.0230 & 0 \\
\hline
\end{tabular}

determining the probability of bit error in digital communication even when the correct variance can be evaluated by independent means.

3.2. Probability of bit error. To evaluate the impact of non-Gaussian noise distribution on the digital signal bit error probability $P_{e}$, the probability of error is computed both with Gaussian and non-Gaussian distribution given by (3.36). For the case of bipolar NRZ signaling, the sampled signal at the FM demodulator output takes values $\pm V$ for some voltage $V$. The sampled output SNR equal to $\left(V / \sigma_{f}\right)^{2}$ is dependent upon the input $\mathrm{SNR}$, the modulation index, and several other factors. In this paper to evaluate the impact of non-Gaussian noise distribution, the probability of error is computed for a given output SNR both for the Gaussian and non-Gaussian distribution of noise both with the same variance. The non-Gaussian noise distribution is parameterized by the input SNR. Figure 3.3 compares the results for the non-Gaussian noise distribution corresponding to 


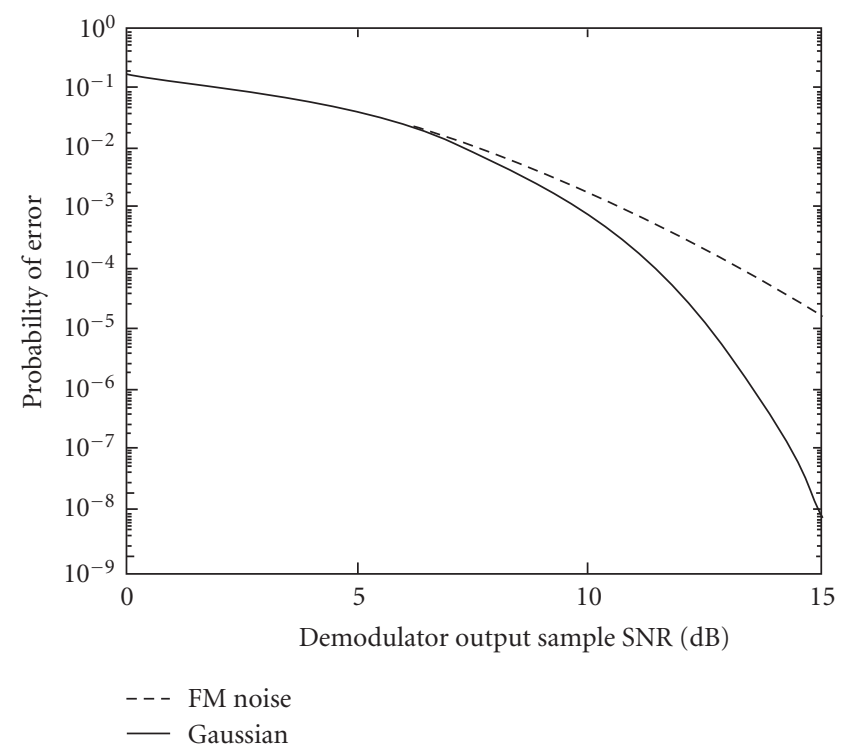

Figure 3.3. Probability of bit error for NRZ signal $\left(\mathrm{SNR}_{i}=12 \mathrm{~dB}\right)$.

input SNR of $12 \mathrm{~dB}$. The figure shows in very clear terms, the difference in $P_{e}$ resulting from the non-Gaussian distribution.

\section{Conclusions}

This paper has presented an exact analysis of the FM demodulator output noise under the assumption of low-modulation index and Gaussian-distributed noise at the demodulator input. It has been shown that for low to medium SNRs the pdf of the FM demodulator output noise differs significantly from the Gaussian pdf. A detection example has been presented to illustrate possible impact of the non-Gaussian noise on the probability of detection error. The derivation of the paper assumed low-modulation index and does not include the effect of the postdemodulation (lowpass) filter on the probability distribution (the effect on the variance is implicitly accounted for in the detection example). For the digital modulation schemes, earlier simulation studies show that the best performance is achieved when the lowpass filter bandwidth is of the order of the IF bandwidth. In such cases the impact of the lowpass filter is expected to be relatively small. A semianalytical approach can be used to evaluate the impact of lowpass filter under more diverse conditions.

\section{References}

[1] L. W. Couch, Digital and Analog Communication Systems, Macmillan, New York, 2000.

[2] I. S. Gradshteyn and I. M. Ryzhik, Table of Integrals, Series, and Products, Academic Press, New York, 1980. 
[3] A. Papoulis, Probability, Random Variables, and Stochastic Processes, McGraw-Hill, New York, 1991.

[4] S. O. Rice, Mathematical analysis of random noise, Selected Papers on Noise and Stochastic Processes (J. L. Doob and L. S. Ornstein, eds.), Dover, New York, 1954.

[5] H. Taub and D. L. Schilling, Principles of Communication Systems, McGraw-Hill, New York, 1986.

Rajendra Kumar: Department of Electrical Engineering, California State University, Long Beach, CA 90840, USA; Communications Systems Department, The Aerospace Corporation, El Segundo, CA 90245-4691, USA

E-mail address: kumar@csulb.edu 


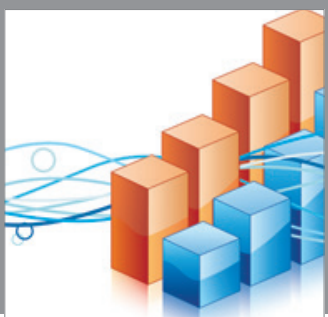

Advances in

Operations Research

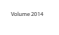

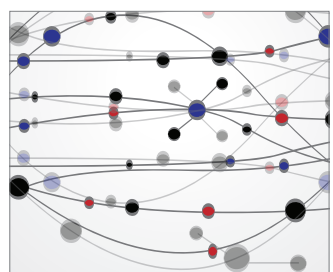

\section{The Scientific} World Journal
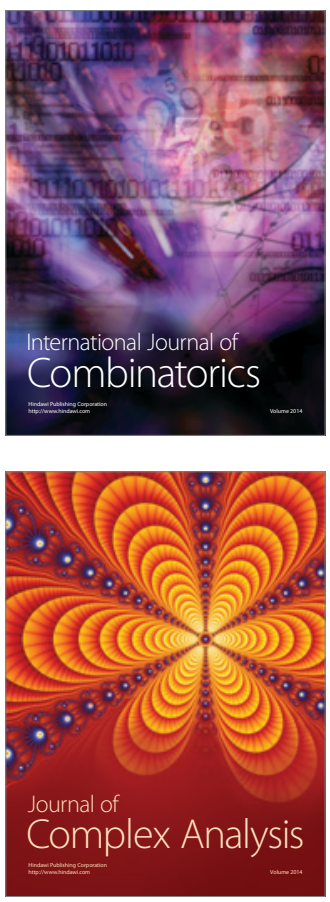

International Journal of

Mathematics and

Mathematical

Sciences
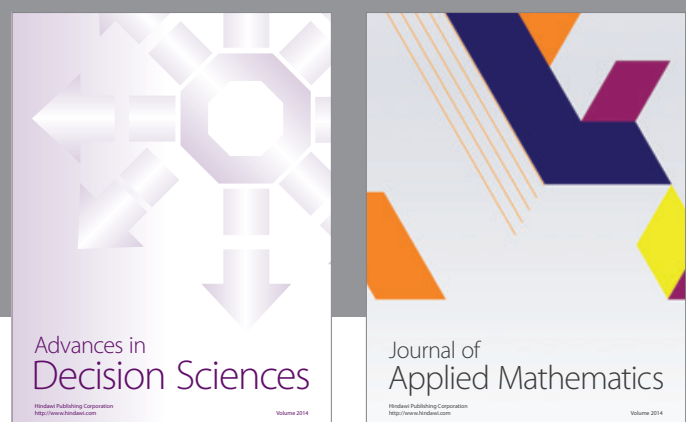

Journal of

Applied Mathematics
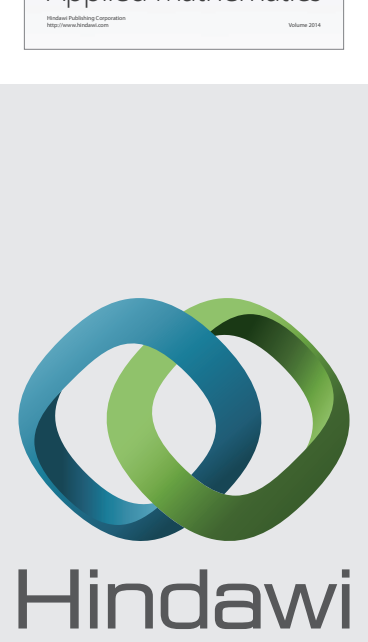

Submit your manuscripts at http://www.hindawi.com
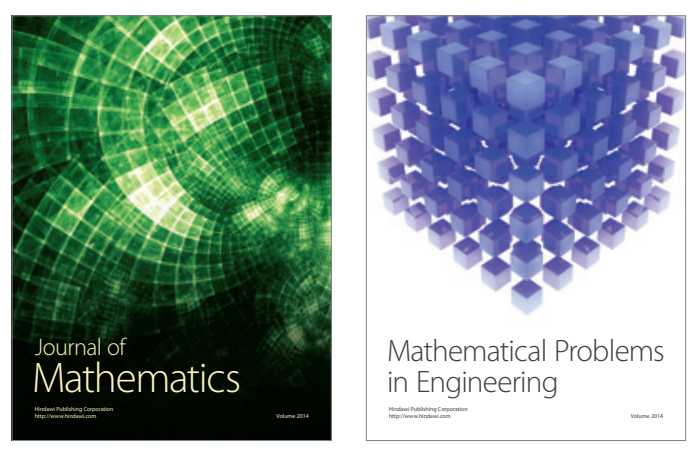

Mathematical Problems in Engineering
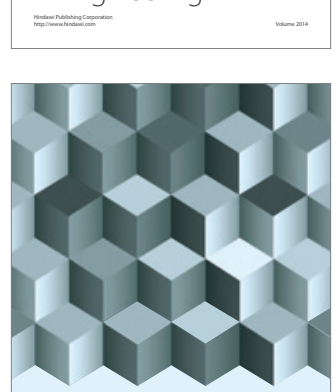

Journal of

Function Spaces
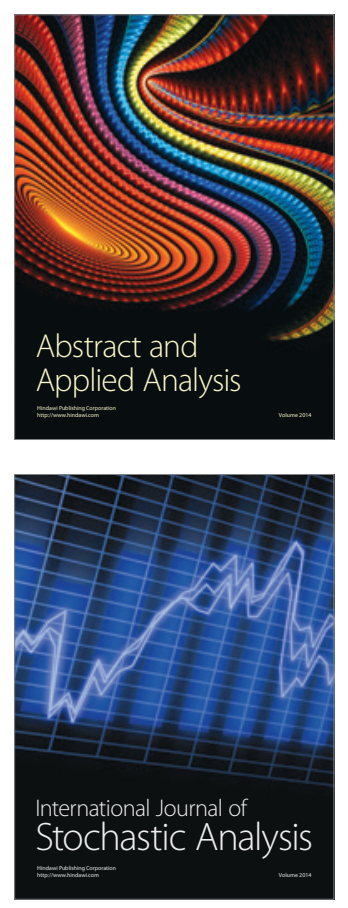

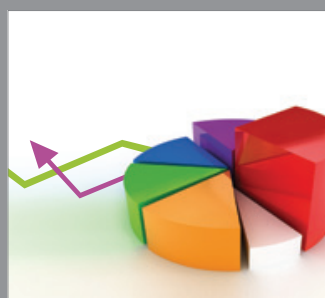

ournal of

Probability and Statistics

Promensencen
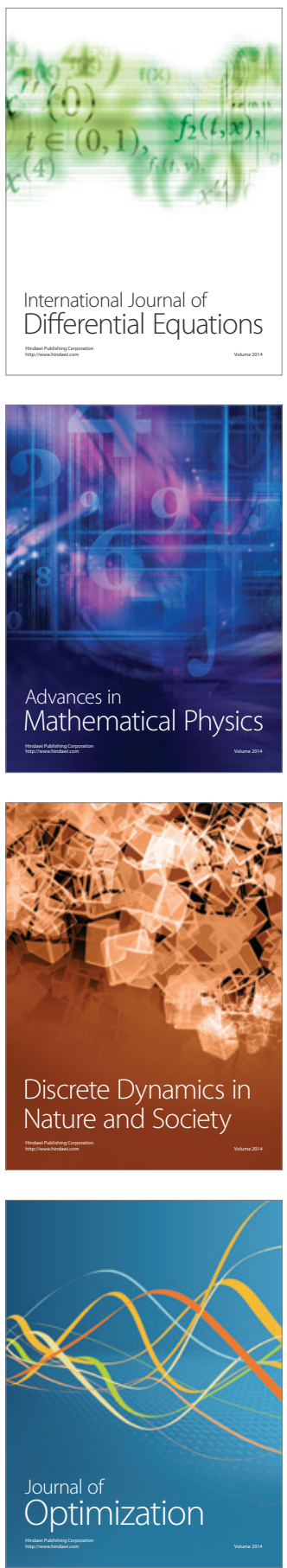